\title{
Evaluación Formativa de la Lengua de los Profesores de Science: un Estudio de Caso
}

\author{
Formative Language Assessment for Bilingual Science Teachers: \\ A Case Study \\ María Ángeles Martín del Pozo ${ }^{a}$, Marta Herrero de la Calle ${ }^{b}$ \\ ${ }^{\mathrm{a}}$ Universidad de Valladolid \\ Correo electrónico: maryange@dlyl.uva.es. \\ ${ }^{\mathrm{b}}$ Universidad de Valladolid
}

\section{RESUMEN}

La educación bilingüe se está implantando de forma cada vez más creciente en los centros de Educación Primaria de España. Entre los muchos retos que se plantean está el de la formación del profesorado. Este artículo propone la evaluación formativa por medio de la observación entre iguales como una posible herramienta para contribuir a la formación del profesorado de educación bilingüe. El trabajo comienza resaltando la relevancia del apoyo lingüístico del profesor al alumno en situaciones de aprendizaje bilingüe. Se contemplan las ventajas y limitaciones de la observación entre iguales como evaluación formativa. De la consideración de ambos surge un instrumento de evaluación formativa que se implementará para la observación de un profesor impartiendo clase de Science en $2^{\circ}$ curso de Educación Primaria. Los resultados de la observación permiten ver el tipo de apoyo más frecuente y orientar al profesor en la mejora de su práctica.

Palabras claves: evaluación formativa, observación entre iguales, formación del profesorado, Aprendizaje Integrado de Contenidos y Lengua Extranjera (AICLE).

\begin{abstract}
Bilingual education is increasingly being implemented in Spanish primary education. Teacher education is one of the numerous challenges that must be faced in this context. This paper suggests formative assessment through peer observation as a possible tool to contribute to bilingual teacher education. The paper begins by highlighting the importance of linguistic support provided by teachers to students in bilingual learning. Then, the advantages and limitations of peer observation as formative assessment are considered. Subsequently, an instrument for formative evaluation is proposed and implemented in the observation of a science teacher working in year two of primary education. Results of the observation show the most frequent types of support and provide guidance for the teacher to improve her practice.
\end{abstract}

Keywords: formative assessment, peer observation, teacher training, Content and Language Integrated Learning (CLIL). 


\section{INTRODUCCIÓN}

El aprendizaje integrado de contenidos y lengua extranjera (AICLE), o su equivalente inglés CLIL (Content and Language Integrated Learning), son denominados popularmente "educación bilingüe". Esta realidad educativa se presenta como el futuro de la educación en muchos países de la Unión Europea y de América Latina. La celeridad con que se ha implantado AICLE ha sobrepasado el ritmo de la formación del profesorado (Coyle, Hood \& Marsh, 2010). Asimismo, esta formación continúa siendo un punto de debate abierto como muestran numerosos estudios de muy diversa índole (Martín del Pozo, 2015; Pérez Cañado, 2014, 2016). El presente trabajo pretende contribuir a la formación del profesorado de AICLE reflexionando sobre el potencial educativo de la evaluación formativa en contextos de observación docente.

Para ello se presenta un instrumento de evaluación debidamente fundamentado en los postulados teóricos de una buena práctica AICLE. Dicho instrumento se implementa para observar a un profesor en una sesión de Science en Educación Primaria y poder ofrecerle feedback descriptivo y evaluativo de un aspecto muy concreto: su uso del apoyo lingüístico. Este estudio de caso centrado en el análisis lingüístico pretende poner de relieve que la observación sistemática entre iguales tiene potencial como evaluación formativa para los docentes en activo (Hamilton, 2012; Harper \& Nicolson 2012).

Las conclusiones establecidas tras el análisis lingüístico de la sesión podrían servir para marcar unas líneas generales respecto a la didáctica de Science. Además, remarcan la necesidad de que los profesores lleven a cabo análisis de sesiones para que sean capaces de detectar las demandas lingüísticas que generan sus sesiones en sus alumnos, porque de este modo podrán seleccionar las formas de apoyo lingüístico más adecuadas para satisfacerlas.

\section{MARCO TEÓRICO}

En el marco teórico que precede a nuestro estudio de caso consideramos dos aspectos. Por una parte las exigencias lingüísticas del aprendizaje de una disciplina no lingüística utilizando una lengua extranjera como vehículo. En segundo lugar, reflexionamos sobre un tipo de evaluación formativa, la observación entre iguales, su potencial y sus carencias.

\subsection{EL APRENDIZAJE INTEGRADO DE CONTENIDOS Y LENGUA EXTRANJERA}

Aunque una de las consecuencias de la celeridad de implantación de AICLE es la carencia de un marco teórico consensuado y evidente (Dalton-Puffer, 2007; Nikula et al., 2016), sí pueden delimitarse algunos aspectos metodológicos indiscutibles de estas nuevas formas de trabajo.

Uno de ellos es que el enfoque AICLE requiere "métodos activos, un manejo cooperativo de la clase y un énfasis especial en todo tipo de comunicación (lingüística, visual y kinestésica)" (Pavesi et al., 2001, p. 121). El presente trabajo va a focalizarse en la importancia y alcance de la comunicación lingüística para una pedagogía AICLE eficaz.

En relación con esto, otra característica del enfoque AICLE hace referencia a la necesidad de herramientas que compensen las necesidades lingüísticas del alumnado (Clegg, 2007; Consejería de Educación de la Junta de Andalucía, 2013). Esta necesidad se 
justifica si se tiene en cuenta que aprender una materia en lengua extranjera implica mayor esfuerzo cognitivo que el mismo proceso en su lengua materna. Las principales habilidades lingüísticas (leer, escribir, escuchar, hablar e interactuar) son en cierto modo automáticas en la lengua materna y los alumnos las usan de manera natural y rutinaria. Los siguientes párrafos profundizan en esta característica y en sus implicaciones didácticas.

En los contextos AICLE, los alumnos están aprendiendo a la vez nuevos contenidos curriculares y una nueva lengua. Es más, la lengua nueva es a su vez el vehículo de aprendizaje mediante el que están aprendiendo esos nuevos conceptos. Si se le pide al alumno que haga esto sin un apoyo lingüístico se está reduciendo su capacidad para aprender. La educación bilingüe no pretende hacer más difícil el aprendizaje, por lo tanto, requiere una pedagogía de apoyo para mantener o incrementar los niveles de aprendizaje de la asignatura curricular cuando los alumnos AICLE no tienen fluidez.

El consultor educativo independiente Clegg (2007) expone que "enseñar asignaturas en una segunda lengua a alumnos que aún no tienen fluidez en el idioma de aprendizaje aumenta las demandas cognitivas que las lecciones generan en ellos" (p. 113). Para mantener la eficacia de aprendizaje en comparación con la clase en lengua materna, los profesores de las asignaturas necesitan por tanto utilizar una pedagogía que, por una parte, compense las carencias lingüísticas y por otra, divida en menor medida los recursos atencionales. La pedagogía propuesta por Clegg (2007) consiste en apoyar a los estudiantes en el uso de habilidades en lengua extranjera dentro de las tareas objeto de aprendizaje. Por este motivo, establece que existen dos pilares en la educación en una segunda lengua: las exigencias lingüísticas y el apoyo lingüístico.

Los profesores de las asignaturas necesitan ser conscientes de las exigencias que sus lecciones generan en las capacidades de sus alumnos en la segunda lengua (L2); y donde las exigencias exceden esas capacidades, ellos deben proporcionar apoyo lingüístico. El apoyo puede ser proporcionado de dos maneras principales con el fin de reducir las exigencias cognitivas de los estudiantes:

1) Por un lado, el maestro puede reducir las exigencias lingüísticas de la tarea, lo que permite a los alumnos atender con mayor eficacia a los conceptos.

2) Por otro lado, pero con menor frecuencia, puede reducir las demandas conceptuales de la tarea, lo que permite a los estudiantes atender a la lengua.

Clegg (2007) advierte de los peligros a los que se ve expuesta la eficacia del aprendizaje y afirma que cuando el apoyo lingüístico es necesario e incluso requerido, pero no es proporcionado, los alumnos pueden aprender menos de lo que aprenderían si estuviesen aprendiendo en su lengua materna. De hecho, sin esta pedagogía de apoyo, no solamente el aprendizaje puede ser menos eficaz, sino que los programas pueden llegar a excluir a alumnos que no pueden satisfacer estas demandas.

\subsection{LA EVALUACIÓN FORMATIVA Y LA OBSERVACIÓN ENTRE IGUALES}

El concepto de "evaluación formativa", entendido en un sentido amplio, conlleva la obtención de feedback temprano, frecuente y encaminado a la mejora (López-Pastor, 2012). Desde la perspectiva del profesor, la evaluación formativa debe servir para una mejora en tres dimensiones: mejorar el proceso de aprendizaje del alumnado, mejorar la propia 
competencia docente, mejorar los procesos de enseñanza-aprendizaje que desarrollamos en las aulas con nuestro alumnado.

El presente artículo desplaza al profesor de su posición de evaluador y lo coloca en la posición del evaluado. En inglés suele utilizarse el término "Peer-observation" para referirse a este tipo de procesos en los que un profesor evalúa a otro profesor. La literatura especializada en este ámbito de la coevaluación se centra con frecuencia en la evaluación entre alumnos (Kearney, Perkins \& Kennedy-Clark, 2016). Se hace necesario avanzar en la coevaluación entre profesores y desarrollar técnicas, procedimientos e instrumentos que la hagan eficaz (Engin \& Priest, 2014; Sullivan et al., 2012). La evaluación entre compañeros profesores suele darse en contextos relacionados con la formación permanente del profesorado o con la investigación educativa. Dicha observación entre iguales y feedback generado se plantean como un mecanismo para el desarrollo profesional (Byrne, Brown \& Challen, 2010; Mortaz Hejri, Mirzazadeh \& Jalili, 2018), también en el ámbito de los docentes de segundas lenguas (Howard \& Donaghue, 2015). En contextos de universidades americanas, Berman (2003) señala cuatro áreas interrelacionadas para evaluar al profesor: presentación y transmisión, diseño del curso, ambiente en el aula (por ejemplo interacción, equidad), aprendizaje del alumno.

Es obvio que esta propuesta es transferible a otros niveles educativos, pues estas cuatro áreas son comunes a toda práctica docente. El trabajo aquí presentado se centra en la primera de estas áreas: la presentación y transmisión, es decir, todos los elementos relacionados con la comunicación. Existen distintas posibilidades de evaluación de esta presentación y transmisión del profesor (Berman, 2003): cuestionarios, evaluación grupal, grabaciones para auto-observación y observación de compañeros.

Según Berman (2003), la observación entre compañeros es un excelente método para mejorar la docencia. Las principales ventajas encontradas en una observación formativa entre iguales son tres. En primer lugar, para el profesor observado un observador cordial puede ser una fuente de información acerca de los contenidos impartidos, sobre comportamientos y dinámica de trabajo. En segundo lugar, esta observación puede servir de pasarela para discutir de manera conjunta algunos problemas docentes. Finalmente, de esta manera se mantiene al profesorado en contacto con la experiencia de ser estudiante. Reiteramos que, aunque Berman (2003) escribe para contextos de educación superior, cualquier nivel educativo puede beneficiarse de estas ventajas de una observación entre iguales.

Las ventajas de esta observación se reiteran en la literatura (Sullivan et al., 2012). Las observaciones para proporcionar a los compañeros un feedback descriptivo de las prácticas de enseñanza y aprendizaje constituyen un medio para mejorar la calidad de la docencia.

No obstante, el profesorado puede considerar la observación entre iguales como una práctica intrusiva, amenazadora y altamente subjetiva. Así lo documenta el estudio de Bell y Mladenovic (2008). Según este estudio, este carácter intrusivo y subjetivo hace que los profesores sean reacios a ser observados por otro compañero o a considerar esta observación como una herramienta de aprendizaje. La intrusividad y amenaza están dentro del ámbito emocional y son, por lo tanto, elementos menos controlables. Por el contario, la objetividad de la evaluación es más controlable. En el mismo estudio, Bell y Mladenovic (2008) presentan este problema y a la vez sugieren una solución: para que la observación entre iguales funcione, según dichos investigadores, se requiere que el feedback no sea crítico y que promueva desarrollo profesional. 
Un modo concreto y eficaz para alcanzar esa observación formativa de manera no subjetiva es el uso de guías o protocolos para observaciones o grabaciones de observaciones. Estas guías o protocolos son en sí mismas tan valiosas que llevan a afirmar que "In fact, a well-designed observation guide constitutes a mini-lesson in what is observable about effective teaching" (Berman, 2003, p. 9). De aquí puede deducirse la conveniencia de diseñar y elaborar guías, protocolos u otros instrumentos para la observación entre iguales.

El presente trabajo propone e implementa un instrumento de observación entre iguales encaminado a ayudar al profesor observado a mejorar su práctica docente. Es decir, se sugiere un instrumento de evaluación formativa de la práctica docente. Todo esto se realiza en un contexto muy específico: la didáctica de una disciplina no lingüística en lengua extranjera; y para evaluar solo una dimensión muy concreta de todo el proceso de enseñanza y aprendizaje en el contexto ya especificado. La dimensión observada es el apoyo lingüístico que el profesor (consciente o inconscientemente) va ofreciendo a sus alumnos al enseñarles contenidos de una materia utilizando como lengua vehicular el inglés. Por lo tanto, los objetivos de este trabajo se resumen así:

1) Proponer un instrumento de observación entre iguales como evaluación formativa para investigar el apoyo lingüístico del profesor en el aula bilingüe.

2) Implementar dicho instrumento en un estudio de caso.

Los apartados previos han contemplado y justificado la conveniencia de la observación entre iguales para el desarrollo profesional, expuestas sus ventajas y sus carencias. El siguiente apartado propone un instrumento para abordar la posible carencia de objetividad en la observación entre iguales. En el apartado tres se fundamenta y se detalla el instrumento para la evaluación formativa que más adelante se-implementa-en el estudio de caso.

\section{INSTRUMENTO PARA LA EVALUACIÓN FORMATIVA DEL PROFESORADO AICLE}

En la primera parte de este apartado se fundamenta la necesidad de observar el apoyo lingüístico que un profesor ofrece a sus alumnos cuando enseña una disciplina no lingüística utilizando una lengua extranjera. En la segunda parte se presenta el instrumento diseñado a partir de los fundamentos anteriores y que va a utilizarse para proporcionar evaluación formativa a los docentes.

\subsection{NECESIDAD DE EVALUAR EL APOYO LINGÜÍSTICO DEL PROFESOR AICLE}

Como ya hemos mencionado en el marco teórico, el aprendizaje simultáneo de lengua extranjera y contenidos aumenta las necesidades lingüísticas en los alumnos, comprometiendo la eficacia del aprendizaje. En las clases de contenidos impartidos en lengua extranjera (por ejemplo, Ciencias naturales en inglés) la comprensión de la lengua acapara la atención del alumno y puede desviarla de los contenidos del área. Esta dificultad se puede compensar recurriendo a la pedagogía del apoyo lingüístico propuesta por Clegg (2007). Dicha pedagogía plantea que el maestro debe proporcionar apoyo lingüístico a los 
estudiantes AICLE. Para estar en condiciones de proveer dicho apoyo, el maestro debe conocer antes las demandas lingüísticas que sus clases generan en los alumnos, además del tipo de apoyo que requiere cada uno, en qué parte de la sesión y en qué grado. Esta información solamente se consigue mediante el análisis de sesiones.

Por todas estas razones, queda fundamentada la importancia de que los maestros lleven a cabo análisis de sesiones. Esta consideración nos lleva a observar directamente el aula y analizar las exigencias lingüísticas generadas por una sesión determinada. El trabajo de análisis se desarrolla en el apartado cinco y se lleva a cabo haciendo uso de los conceptos teóricos expuestos en esta primera parte.

\subsection{INSTRUMENTO PARA LA OBSERVACIÓN}

En el apartado sobre la observación entre iguales con fines de evaluación formativa afirmábamos que uno de los principales riesgos es caer en la subjetividad. Para evitarlo, Berman (2003) sugería el uso de guías y protocolos. Con el fin de contribuir a esta sistematicidad, proponemos una planilla para la observación de sesiones. La plantilla (Tabla 1) recoge elementos del apoyo lingüístico, cuya conveniencia se ha justificado en el apartado precedente.

Tabla 1. Instrumento para la observación de sesiones

\begin{tabular}{|l|l|l|l|}
\hline \multirow{5}{*}{ Tarea específica de apoyo } \\
lingüístico & Tablas de sustitución & Actividad & Comentarios \\
\hline & Listas de vocabulario & & \\
\hline & Uso de modelos & & \\
\hline & Explicación gramatical & & \\
\hline & otros & & \\
\hline \multirow{5}{*}{ Elementos visuales } & Gráfico & & \\
\hline & Diagrama & & \\
\hline & Objetos reales & & \\
\hline & Flash cards & & \\
\hline & Mímica & & \\
\hline \multirow{5}{*}{ Forma de interacción } & Toda la clase & & \\
\hline & Grupo & & \\
\hline & Parejas & & \\
\hline & Trabajo individual & & \\
\hline
\end{tabular}




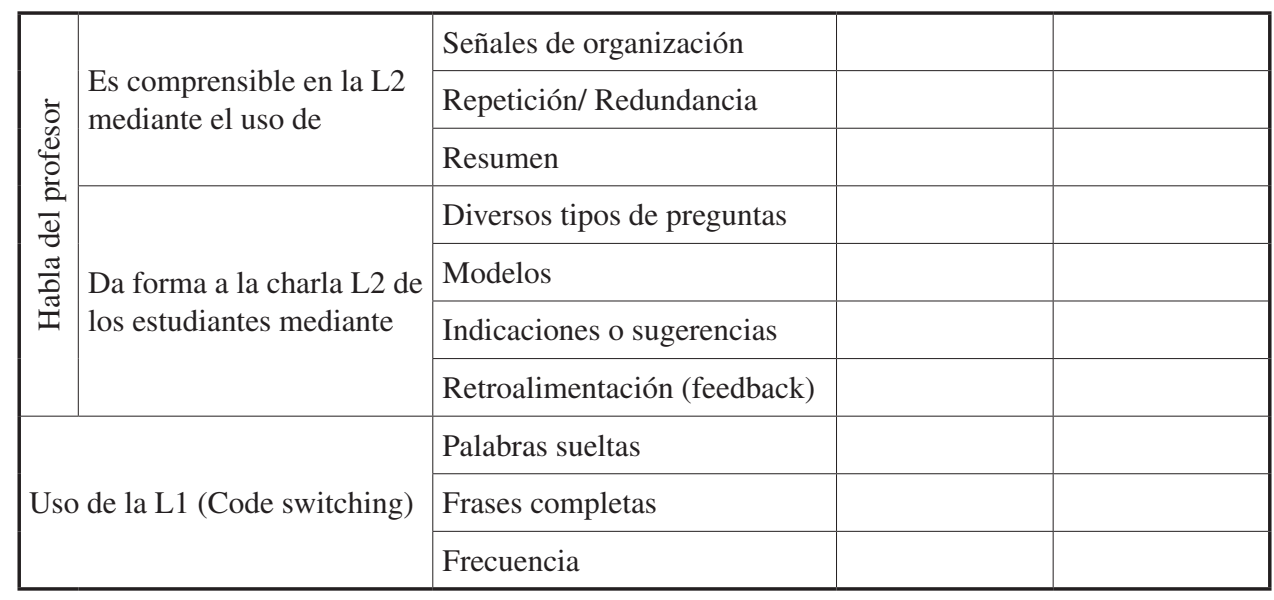

Fuente. Elaboración propia basada en el modelo teórico de Clegg (2007).

\section{CONTEXTO}

Las disciplinas no lingüísticas más comúnmente implicadas en proyectos bilingües de Educación Primaria son las de Conocimiento del Medio Natural, Social y Cultural, seguidas de las materias de carácter artístico (Plástica y Música) o de Educación Física. Este estudio de caso elige la asignatura Science. Science es una de las tres áreas fundamentales del currículo inglés (English, Maths and Science). En el contexto del que estamos hablando, los colegios bilingües españoles, se entiende por Science la asignatura donde se enseñan de forma integrada las áreas de Lengua Extranjera (Inglés) y Conocimiento del Medio Natural, Social y Cultural.

La enseñanza de las Ciencias ofrece una comunicación en contexto gracias a imágenes, mapas o gráficos, por lo que es una de las disciplinas que mejor se prestan a ser impartidas en L2 aun cuando el nivel de competencia lingüística del alumnado sea muy básico. Por otro lado, es relevante destacar la práctica inexistencia de bibliografía referente a la didáctica de Science. Por ello, este trabajo puede ser también una aportación en dicha línea.

Este estudio de caso ha sido realizado en un centro urbano de titularidad pública, dependiente de la Consejería de Educación de la Junta de Castilla y León que imparte los niveles de Educación Infantil y Educación Primaria. El acceso de las investigadoras al campo se debe a la estancia de una de ellas en dicho centro durante el segundo periodo de prácticas del Grado de Maestro en Educación Primaria. Este centro fue uno de los 43 centros pioneros en la implantación del Proyecto Bilingüe del M.E.C.D./British Council ${ }^{1}$.

Los datos se recogieron en un grupo de $2^{\circ}$ curso compuesto por 25 alumnos cuyas edades están comprendidas entre los 7-8 años. Este es su $2^{\circ}$ año de aprendizaje de Science (Conocimiento del Medio en Inglés como lengua de aprendizaje).

Con los correspondientes permisos y tomadas las medidas que se requerían, se grabaron en video varias sesiones. Las grabaciones permiten atender a aspectos que mediante la mera observación podrían pasar desapercibidos (por ejemplo, el lenguaje no verbal o el

El Proyecto Bilingüe del M.E.C.D./British Council nació en 1996 como un experimento único dentro del Sistema Educativo Estatal Español. 
uso de recursos materiales). Se ha seleccionado una sesión determinada debido al interés que encierra desde el punto de vista del objeto de esta investigación. En dicha sesión se da importancia al uso de la lengua, recreando situaciones verosímiles del aprendizaje de las Ciencias y trabajando de forma contextualizada.

El segundo paso consistió en la transcripción de dicha grabación para poder proceder al análisis cualitativo de los datos según las categorías recogidas en el instrumento. La Tabla 2 resume las circunstancias de la recogida de datos y la Tabla 3 presenta el orden temporal de actividades realizadas en la sesión.

Tabla 2. Información relevante referente a la recogida de datos

\begin{tabular}{|l|l|}
\hline Tipo de grabación & Video \\
\hline Tiempo de grabación & $56^{\prime} 29^{\prime}$ \\
\hline Número de palabras & 5550 aprox. \\
\hline Maestra que imparte la clase & Tutora del grupo 2 ${ }^{\circ}$ A \\
\hline Área & Science \\
\hline Tema de la clase & "Materials" ("Los materiales") \\
\hline Nivel & $2^{\circ}$ de Educación Primaria \\
\hline Número de alumnos & 25 alumnos \\
\hline
\end{tabular}

Tabla 3. Cronograma de la clase analizada

\begin{tabular}{|l|c|}
\hline \multicolumn{1}{|c|}{ Partes en que se divide la sesión } & Tiempos \\
\hline Rutinas: fecha y tiempo atmosférico & $00: 00-03: 33$ \\
\hline Spelling test (Examen de vocabulario) & $03: 34-11: 44$ \\
\hline Repaso de los conceptos referentes a los materiales & $11: 45-13: 16$ \\
\hline Actividad 1. Recordar los materiales y su posición & $13: 17-17-23$ \\
\hline Actividad 2. Listening Cosmo's house (Comprensión oral) & $17: 24-30: 44$ \\
\hline Actividad 3. Lectura de la teoría & $30: 45-33: 04$ \\
\hline Actividad 4. Completar una tabla sobre las características de los materiales & $33: 05-43: 46$ \\
\hline Actividad 5. Conceptos: verbos del cambio de forma de la materia & $43: 47-51: 30$ \\
\hline Recoger el material y ponerse en fila para salir & $51: 31-56: 29$ \\
\hline
\end{tabular}

\section{ANÁLISIS DE LA SESIÓN CON EL INSTRUMENTO PARA LA EVALUACIÓN FORMATIVA}

El estudio realizado es de tipo descriptivo, empírico y no-experimental. El fin de esta observación es obtener una visión general de las demandas linguísticas de esta clase y 
de las características del apoyo lingüístico facilitado por la maestra para esta sesión en particular. Los datos se analizan en orden cronológico de la sesión.

La sesión comienza con las rutinas de fecha y tiempo atmosférico, muy importantes al trabajar con este grupo de edad. El papel de la maestra consiste en ser guía y el principal apoyo que proporciona es intervenir para dar forma a las intervenciones de los alumnos mediante preguntas que constituyen indicaciones o sugerencias para éstos. Estas preguntas fomentan el diálogo de los alumnos en torno a las rutinas y les permiten hacer pequeñas aportaciones de expresión oral.

La segunda actividad consiste en un examen de vocabulario (spelling test). La maestra dicta y los alumnos escriben 10 conceptos, cuya grafía han memorizado durante el fin de semana. A pesar de que los contenidos conceptuales evaluados en este spelling test no tienen nada que ver con los de la sesión analizada, pues pertenecen a un tema trabajado con anterioridad en el aula, nos detenemos en esta parte de la sesión por el valor que tienen estas pruebas en el aprendizaje de terminología de Ciencias en una lengua extranjera.

Consideramos relevante reafirmar que las técnicas de evaluación de conocimientos de la asignatura que se han adquirido a través de L2 no son únicamente pruebas de evaluación cuya utilidad se reduzca a registrar e informar a la maestra del aprendizaje obtenido por el alumno. La realización de este tipo de exámenes de vocabulario tiene una justificación añadida desde el punto de vista del apoyo lingüístico, como bien propone John Clegg (2007). A través de la memorización de la escritura de una serie de términos, los alumnos están trabajando por sí mismos para reducir sus necesidades lingüísticas. De este modo la maestra consigue paliar en cierto grado las demandas lingüísticas que el aprendizaje de contenidos curriculares de Ciencias en inglés genera de forma constante.

Antes de comenzar a realizar actividades relacionadas con "los materiales", el contenido de la clase observada, se lleva a cabo un repaso de los conceptos referentes a los materiales. La maestra recurre a las tarjetas de vocabulario (flashcards) como apoyo lingüístico. Estas tarjetas proporcionadas por la editorial del libro de texto con dibujos a todo color ilustran el vocabulario y los conceptos clave (Figura 1).

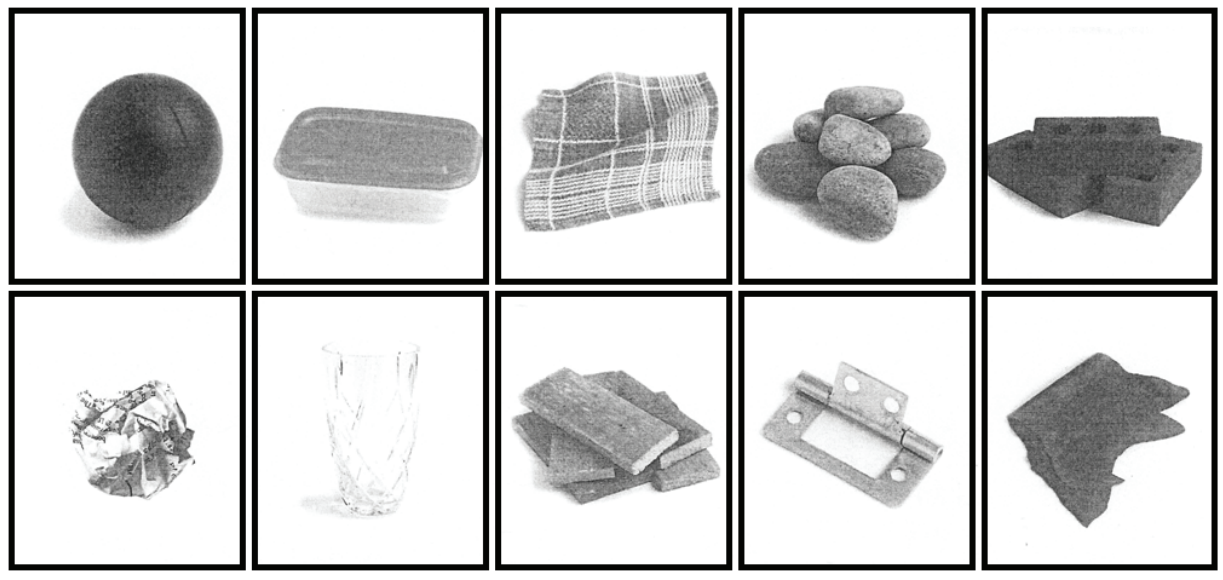

Figura 1. Tarjetas de vocabulario (flashcards) relativas a los materiales 
Su principal finalidad consiste en ofrecer un apoyo visual para el aprendizaje de dicho vocabulario. Consideramos que, de este modo, se está proporcionando un importante estímulo visual que ayuda a la comprensión y mantiene la atención de los alumnos enfocada en el concepto, consiguiendo un aprendizaje a nivel léxico semántico basado en la asociación de conceptos a imágenes.

\section{Actividad 1: Repaso de los materiales}

El repaso de los conceptos referentes a los materiales es dirigido por la maestra sin apenas utilizar lenguaje de gestión del aula. Simplemente organiza las intervenciones de los alumnos nombrando a quien quiere que diga en voz alta el nombre del material de la tarjeta de vocabulario que ella señala con el dedo. Inmediatamente después de que el alumno elegido designe el material, la maestra proporciona apoyo lingüístico, esta vez lo consigue al repetir de nuevo el término. Así está promoviendo la adquisición del nivel fonológico de la lengua mediante la repetición redundante de los conceptos, sirviendo de modelo de pronunciación.

\section{Actividad 2: Listening Cosmo’s house (Comprensión oral)}

La segunda actividad trabaja la comprensión oral mediante una audición de la historia Cosmo's house. Los personajes de la historia son dos niños que van a construir una casa para su mascota, el camaleón Cosmo. Los conceptos y el lenguaje a aprender se presentan de forma contextualizada, en una situación real, lo cual es más favorable en el aprendizaje de la lengua que presentar el vocabulario de forma aislada. El otro tipo de apoyo lingüístico utilizado en esta ocasión consiste en una historia por imágenes secuenciada en forma de seis viñetas de cómic (Figura 2). Las imágenes sirven de apoyo para la comprensión oral. Al mismo tiempo, de nuevo el apoyo visual sirve de soporte en el aprendizaje de los contenidos conceptuales, pues los alumnos disponen de imágenes a las que asociar el vocabulario que escuchan.

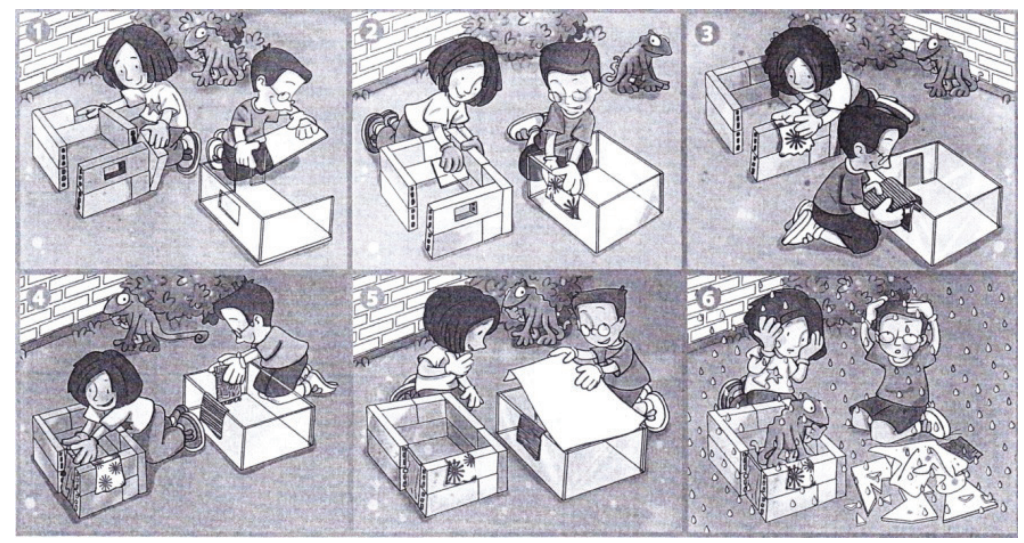

Figura 2. Viñetas para apoyar la audición 
Después de escuchar, la maestra detiene la grabación y elige un niño que repite en voz alta y para toda la clase lo que acaba de escuchar, por último, lo traduce al español. El alumno lleva a cabo en primer lugar la tarea de comprensión oral. Mediante el último paso de traducción a la lengua materna, se comprueba de alguna manera si el alumno ha sido verdaderamente capaz de construir el significado de lo que ha percibido. Por otro lado, al repetir lo que acaba de escuchar trabaja la expresión oral, sobre todo en cuestión de claridad y fluidez articulatoria, así como de fuerza expresiva.

Consideramos la propia grabación como una forma de apoyo lingüístico en esta actividad, porque sirve de modelo del nivel fonológico de la lengua. Lo que los alumnos escuchan se convierte en el punto de referencia para imitarlo o reproducirlo cuando tienen que repetirlo. A su vez, las intervenciones de los personajes constituyen modelos morfosintácticos de construcción de frases sencillas.

En esta última parte de la sesión la maestra recurre a una nueva forma de proporcionar apoyo lingüístico en la enseñanza de asignaturas en L2: la traducción a la lengua materna del alumno. A pesar de las reticencias generales respecto al uso de este recurso, Clegg (2007) recomienda, como una de las principales características de la pedagogía del apoyo lingüístico, "el uso, según el criterio del docente, de la L1 en la lección de L2-medio" (p. 115).

Por otro lado, Pavesi et al. (2001) afirman:

El cambio de código es una estrategia natural en la comunicación, y los profesores deberían permitirlo, sobre todo en las primeras fases de AICLE. Esto no significa que el profesor deba utilizar la traducción para resolver cualquier dificultad o malentendido. Se deben utilizar otras estrategias de comunicación, por ejemplo una nueva formulación, simplificación y ejemplificación, recurriendo a la traducción solamente en última instancia. (pp. 20-21).

A la hora de traducir del inglés al español, se generan ciertas exigencias lingüísticas en los alumnos. En la mayoría de ocasiones estas demandas corresponden al desconocimiento de algunas palabras o, como ocurre en el caso particular que se presenta a continuación, a que los contenidos lingüísticos objeto de estudio aún no están afianzados.

Teacher: Wood is hard and rigid. Cristian!

Cristian: Wood is hard and rigid. La madera es dura y...

Teacher: Rigid is the contrary to bendy. When we can not bend something we say it's rigid. ... Do you remember "bones are hard and rigid"? Daniel!

Daniel: ¡Rígido!

Teacher: Right! ¡Rígido! La madera es dura y rígida.

En este ejemplo vemos como la maestra ofrece apoyo de dos formas diferentes. Por un lado, recurre al antónimo del concepto a traducir, de este modo lo explica mediante la idea opuesta, incidiendo en que se trata de lo contrario (ej.: "Rígido es lo contrario de flexible. Cuando no podemos doblar algo decimos que es rígido").

Por otro lado, la maestra ofrece un ejemplo en el que el concepto en cuestión se presenta de forma contextualizada, enmarcado en una situación de aprendizaje que el alumnado conoce, pues se trata de contenidos que han sido trabajados en el aula con anterioridad (“¿Recordáis? Los huesos son duros y rígidos”). En este caso el apoyo lingüístico 
proporcionado ha sido efectivo y el alumno ha sido capaz de satisfacer sus demandas lingüísticas sin necesidad de que la maestra haya recurrido a la traducción literal.

En ocasiones tienen lugar errores conceptuales a nivel léxico semántico debidos a la similitud entre algunas palabras. En el ejemplo siguiente se observan las dificultades del alumnado hasta llegar a la correcta traducción del concepto "fabric" (tela). Así cometen los errores de identificar esa palabra con los conceptos "fábrica", en español, y "bricks" (ladrillos), en inglés. Se podría decir que, en el primer caso, "fabric" (inglés) y "fábrica" (español) son una pareja de palabras conocidas como "false friends", pues se trata de parónimos, vocablos de dos lenguas diferentes que se asemejan en su forma y sonido pero que difieren significativamente en su significado.

El tratamiento que la maestra hace del error consiste en llamar la atención sobre el mismo y formular una serie de preguntas dirigidas a toda la clase que promuevan la reflexión por parte de los alumnos. Así guía la corrección del error para que sean los propios estudiantes quienes la lleven a cabo mediante indicaciones o sugerencias. Este es un claro ejemplo de que el estilo de intervención de la maestra proporciona apoyo lingüístico en sí mismo, como propone Clegg (2007) y como veíamos en el marco teórico de este trabajo.

\section{Actividad 3: Lectura de la teoría}

La tercera actividad se centra en tres oraciones que aparecen en un recuadro del libro de texto (Figura 3) y que engloban y relacionan entre sí los tres grupos de conceptos en torno a los que se articula la sesión: partes de la casa, materiales y propiedades de los materiales. La maestra pide que un alumno lea la primera afirmación. Éste la lee, luego la traduce al español y finalmente la maestra reproduce el archivo de audio correspondiente a esa oración.

$$
\begin{aligned}
& \text { Some materials are better for a purpose than others. } \\
& \text { Windows are made of glass because glass is transparent. } \\
& \text { Houses are made from bricks because bricks are rigid. }
\end{aligned}
$$

Figura 3. Libro de texto

El apoyo, ya proporcionado por el propio libro de texto, es visual, puesto que los conceptos que suponen la carga de contenido aparecen marcados y diferenciados visualmente mediante el color de la letra. Valoramos positivamente la función de este método al dirigir los recursos atencionales de los alumnos hacia los conceptos lingüísticos.

\section{Actividad 4: Completar una tabla sobre las características de los materiales}

Antes de comenzar a completar la tabla (Figura 4), la maestra vuelve a hacer un breve repaso de los conceptos correspondientes a las características de los materiales. 


\begin{tabular}{|l|c|c|c|c|c|}
\hline & Transparent & Bendy & Rigid & Soft & Hard \\
\hline wood & no & & & \\
\hline
\end{tabular}

Figura 4. Tabla de las características de los materiales

De nuevo surgen dudas conceptuales. La maestra utiliza en esta ocasión, además de la redundante repetición de los conceptos en el discurso, otros dos tipos de apoyo lingüístico diferentes. En primer lugar, intenta elicitar términos mediante el lenguaje no verbal, haciendo mímica y gestos que representan el verbo que quiere que el alumno diga. El uso de lenguaje no verbal no es contemplado por Clegg (2007) en su teoría como una forma de satisfacer demandas lingüísticas. Sin embargo, estimamos que es igual, o incluso más eficaz que el apoyo lingüístico que proporcionan otros materiales de carácter visual, por eso lo hemos incluido en nuestro instrumento de observación (Tabla 1).

La otra manera de ofrecer apoyo lingüístico en este momento es recurriendo a objetos con los que poder ejemplificar ciertas propiedades de los materiales con los que están fabricados. El uso de objetos tampoco está incluido en la propuesta inicial de Clegg (2007), pero desde nuestro punto de vista se trata de otra forma de apoyo visual. Podríamos decir que es un apoyo más completo, sobre todo en lo que compete a las Ciencias, pues incluye la posibilidad de manipular los objetos y fomenta el aprendizaje vivencial.

Con el fin de trabajar la expresión oral, la maestra plantea que la tabla se complete en común en el aula mediante preguntas y respuestas encadenadas. La actividad engloba así las habilidades lingüísticas de leer, escuchar, hablar y escribir, así como los niveles fonológico, léxico sintáctico y morfosintáctico de la lengua. Podría decirse que rellenar la tabla constituye una tarea globalizadora en cuanto a la lengua, la cual se utiliza para las funciones de clasificar y solicitar y dar información.

Un ejemplo de aprendizaje a nivel morfosintáctico es que los alumnos están practicando la construcción de oraciones interrogativas utilizando el presente simple. La estructura gramatical de las preguntas que los alumnos deben formular es: "Is the (material) (propertie)?” (“¿Es el/la (material) (propiedad)?”). Ej.: Is the wood transparent? (¿Es la madera transparente?).

\section{Actividad 5: Conceptos: verbos del cambio de forma de la materia}

Los términos y los contenidos resultan nuevos para los alumnos. La maestra lo presenta como "cosas que podemos hacer con los materiales". Se trata de acciones que las personas podemos efectuar con la materia, por lo tanto hablamos de verbos. Una forma de apoyo lingüístico en la enseñanza-aprendizaje de los verbos en una lengua extranjera es mediante 
mímica, expresando acciones por medio de gestos. La maestra representa los verbos objeto de aprendizaje, en principio a través del lenguaje no verbal, únicamente mediante gestos realizados con sus manos.

Debido a que las demandas lingüísticas son persistentes en los alumnos y la mímica parece no ser suficiente, decide ayudarse de algunos objetos cotidianos presentes en el aula para proporcionar el apoyo lingüístico requerido (un trocito de algodón, una cuerda para saltar, un lápiz flexible y un lápiz común). El libro de texto proporciona a su vez un apoyo visual, pues aporta gráficos que representan las maneras en que podemos cambiar la forma de los materiales (Figura 5). No obstante, la mímica y el uso de objetos constituyen un mejor apoyo porque permiten al alumnado un acercamiento más fiel a la realidad que representan los conceptos estudiados.

\section{The shape of some materials can be changed by bending, twisting, stretching or squashing them.}

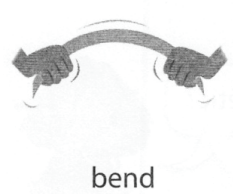

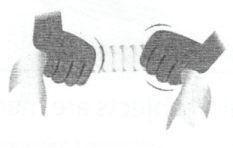

twist

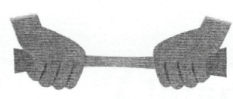

stretch

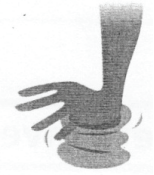

squash

Figura 5. Ilustración de las maneras en que podemos cambiar la forma de los materiales

Con el fin de que el aprendizaje tenga un carácter vivencial, la maestra anima a algunos alumnos a que salgan a la pizarra a experimentar por sí mismos los cambios de forma en los distintos materiales. Como se trata de aprendizaje de Ciencias, los alumnos razonan en base a la experimentación.

Al mismo tiempo que la maestra utiliza gestos y objetos como apoyo lingüístico, igualmente adquiere un papel importante en esta actividad el uso del lenguaje verbal con el fin de elicitar los términos en los alumnos. La maestra utiliza un estilo de intervención fácilmente comprensible en la L2 mediante expresiones y preguntas que constituyen indicaciones o sugerencias para dar forma a la charla de los alumnos (Who can guess the meaning?... You don't have to know it, right? But you can guess!"; "Dar vueltas... in another way? Just another word... to do this (vuelve a retorcer el trocito de algodón)"; "¡Tirar de ello! And... how do you say that? Mario!”.). La función de estas intervenciones reside en guiar la actividad y suscitar las intervenciones de los niños en la búsqueda de la traducción al español más aproximada de los términos en cuestión.

La estructura gramatical de la pregunta que la maestra formula a los alumnos más frecuentemente es: "Can we (way in which the shape of some objects can be changed) (material or object)?” (“¿Podemos (modo en que puede ser cambiada la forma de algunos objetos) (material u objeto)?"). Ej.: Can we twist cotton? Observamos que la maestra no enseña gramática de forma explícita. Pensamos que esto puede deberse al nivel de los alumnos, todavía temprano para llevar a cabo amplias explicaciones gramaticales. Sin embargo, el método de apoyo lingüístico utilizado por la maestra para suplir la ausencia 
de explicaciones gramaticales y, a su vez, satisfacer las demandas lingüísticas que estas estructuras puedan generar, consiste en hacer un uso redundante de ellas. Las intervenciones de la maestra sirven como modelo por repetición.

\section{RESULTADOS}

La Tabla 4 resume el papel de la maestra. La última columna muestra el tipo de apoyo lingüístico que la maestra propone y lleva a cabo para ayudar a sus alumnos y que ha sido objeto de análisis detallado en los párrafos precedentes.

Tabla 4. Resumen del análisis

\begin{tabular}{|c|c|c|}
\hline Partes de la sesión & Acciones de la maestra & $\begin{array}{l}\text { Formas de apoyo lingüístico } \\
\text { (maestra y recursos) }\end{array}$ \\
\hline $\begin{array}{l}\text { Rutinas: fecha y tiempo } \\
\text { atmosférico. }\end{array}$ & $\begin{array}{l}\text { Guía las rutinas mediante } \\
\text { preguntas. }\end{array}$ & $\begin{array}{l}\text { Preguntas que constituyen } \\
\text { indicaciones o sugerencias. }\end{array}$ \\
\hline $\begin{array}{l}\text { Spelling test (Examen de } \\
\text { vocabulario). }\end{array}$ & Dicta los conceptos. & $\begin{array}{l}\text { El propio examen de } \\
\text { vocabulario. }\end{array}$ \\
\hline $\begin{array}{l}\text { Repaso de los conceptos } \\
\text { referentes a los materiales. }\end{array}$ & $\begin{array}{l}\text { Organiza las intervenciones de } \\
\text { los alumnos. }\end{array}$ & $\begin{array}{l}\text { Tarjetas de vocabulario } \\
\text { (flashcards). } \\
\text { Repetición redundante de } \\
\text { conceptos. }\end{array}$ \\
\hline $\begin{array}{l}\text { Actividad 1: } \\
\text { Recordar los materiales y su } \\
\text { posición. }\end{array}$ & $\begin{array}{l}\text { Explica la actividad y guía el } \\
\text { desarrollo de ésta. }\end{array}$ & $\begin{array}{l}\text { Tarjetas de vocabulario } \\
\text { (flashcards). }\end{array}$ \\
\hline $\begin{array}{l}\text { Actividad 2: } \\
\text { Listening Cosmo's house } \\
\text { (Comprensión oral). }\end{array}$ & $\begin{array}{l}\text { Organiza las intervenciones de } \\
\text { los alumnos y aclara dudas } \\
\text { conceptuales. }\end{array}$ & $\begin{array}{l}\text { Grabación (como modelo). } \\
\text { Historia por imágenes del libro } \\
\text { de texto. } \\
\text { Traducción. } \\
\text { Señales de organización. }\end{array}$ \\
\hline $\begin{array}{l}\text { Actividad 3: } \\
\text { Lectura de la teoría. }\end{array}$ & Guía la traducción de la teoría. & $\begin{array}{l}\text { Grabación (como modelo). } \\
\text { Libro de texto (input de } \\
\text { contenidos). } \\
\text { Traducción. }\end{array}$ \\
\hline $\begin{array}{l}\text { Actividad 4: } \\
\text { Completar una tabla sobre las } \\
\text { características de los materiales. }\end{array}$ & $\begin{array}{l}\text { Explica la actividad y los } \\
\text { conceptos y organiza las } \\
\text { intervenciones. }\end{array}$ & $\begin{array}{l}\text { Tabla del libro de texto. } \\
\text { Redundancia en el discurso de } \\
\text { la maestra. }\end{array}$ \\
\hline $\begin{array}{l}\text { Actividad 5: } \\
\text { Conceptos: verbos del cambio } \\
\text { de forma de la materia. }\end{array}$ & $\begin{array}{l}\text { Explica los conceptos gracias a } \\
\text { objetos y mediante mímica. }\end{array}$ & $\begin{array}{l}\text { Objetos cotidianos. } \\
\text { Gráficos del libro de texto } \\
\text { (input de contenidos). } \\
\text { Preguntas que constituyen } \\
\text { indicaciones o sugerencias. }\end{array}$ \\
\hline
\end{tabular}


Según lo observado, los apoyos predominantes son los visuales (tarjetas de vocabulario, mímica), la repetición /redundancia de lo dicho y la traducción al español. De la misma manera, el propio libro de texto con sus recursos propios (ilustraciones que contiene, audio, actividades) es un apoyo para la maestra no solo en lo que respecta a lengua, sino también al contenido. No es sorprendente que la maestra opte por este tipo de apoyo, que puede ser calificado como propicio para la edad de los niños a los que iba dirigida la sesión (7-8 años).

El uso del instrumento presentado en la Tabla 1 para observar a esta maestra ha permitido una reflexión sobre qué recursos /estrategias está empleando de manera inconsciente. Esta observación proporciona así información sobre la práctica docente: estrategias a las que se recurre y estrategias a las que no se recurre. Si estas últimas se deben no a la no necesidad de esa estrategia determinada, sino a un desconocimiento o falta de aprecio de ella, la evaluación formativa permite que el profesor sea más consciente de esta estrategia y de que esté recurriendo a ella. Por ejemplo, la sesión evaluada no incluye ninguna explicación gramatical. Esto puede deberse a que la propia sesión no requiere esta demanda lingüística o a que el profesor no haya abordado esto por los motivos que sean (preferencias personales sobre las aproximaciones a la didáctica de la gramática, desconocimiento de una forma de trabajo de la gramática con alumnos tan jóvenes, etc.) Lo relevante es que después del análisis de la sesión y de la evaluación la maestra puede reflexionar sobre su propio comportamiento en el aspecto evaluado: el apoyo lingüístico.

\section{CONCLUSIONES}

Las conclusiones a las que nos dirige este estudio de caso pueden agruparse en dos ámbitos. El primero de ellos es el potencial de la observación entre iguales como evaluación formativa para mejora de la práctica docente. El segundo ámbito es el de la didáctica de las Ciencias y otras disciplinas no lingüísticas mediante una lengua extranjera.

La primera conclusión que afecta a la observación entre iguales es el papel clave del instrumento de observación evaluación. Decíamos que uno de los objetivos de este trabajo era proponer un instrumento de observación entre iguales como evaluación formativa para investigar el apoyo lingüístico del profesor en el aula bilingüe. Cuando se observa a compañeros de la misma disciplina es difícil desgajar el contenido y las cuestiones meramente relacionadas con la comunicación y presentación (Berman, 2003). Un compañero de la misma área puede quedarse en aspectos de contenido. Por eso, si se desea un feedback sobre otras cuestiones (como en el caso estudiado) lo aconsejable es ser observado por un compañero de otra área de conocimiento. Un instrumento de evaluación centrado en los elementos lingüísticos como el aquí propuesto e implementado podría ser utilizado por todos sin caer en esa limitación que Berman (2003) preveía. Como puede verse en el apartado de resultados, los comentarios se refieren solo al elemento investigado (el apoyo lingüístico otorgado por la maestra). Este instrumento tiene potencial para ser válido en la evaluación formativa de otros docentes en el aula bilingüe, por ejemplo de docentes que impartan Social Science.

Una segunda conclusión vinculada a la evaluación formativa es que, sin duda, experiencias como esta pueden hacer reflexionar al profesor AICLE sobre su práctica docente de manera sistemática. En primer lugar, el profesor obtiene feedback sobre la 
cantidad de apoyo lingüístico que facilita a sus alumnos y en segundo lugar sobre el tipo y calidad de éste.

Una consecuencia de esto, y a su vez tercera conclusión, es el aprendizaje para el observador. Esta forma de evaluación formativa encierra potencial no solo para el observado. Egin \& Priest (2014, p. 4) invitan al observador a cuestionarse, entre otros "¿Qué han aprendido los participantes de la observación de un compañero?; ¿Hasta qué punto la observación entre iguales ha generado una reflexión crítica?; ¿Qué repercusión tiene en la docencia?" De esta manera, la evaluación formativa es fuente de aprendizaje y herramienta para una reflexión crítica por parte de ambos, el observado y el observador.

El segundo de los ámbitos de las conclusiones de este trabajo es la didáctica de las disciplinas no lingüísticas en una lengua. Ya se ha comentado la práctica inexistencia de bibliografía referente a la didáctica de Science. Se recalca desde este estudio la necesidad de investigar y escribir sobre el tema, con el fin de aportar unas sólidas directrices generales a esta didáctica.

Nos gustaría poner punto final a este trabajo recomendando a los docentes de centros bilingües la realización de observaciones y de análisis lingüísticos de sus clases y proponiendo a la comunidad de investigadores educativos que dirijan su interés hacia este tema. Como recoge la siguiente cita, los profesores bien formados son la clave para una educación bilingüe de calidad:

The success of bilingual education depends on the daily work carried out by teachers in the classroom. Thus, if politicians want a program to succeed they will have to look carefully at ways in which teachers can be supported in their goal of bringing to life this pedagogic innovation. Teachers need time for reflection and training; they need to be provided with materials and other types of resources that make their teaching task easier. (Halbach, 2009, p. 25).

Si un docente reflexiona sobre lo que hace (como aquí se propone), es muy probable que mejore su práctica (Berman, 2003). Los observadores que proveen al profesor de un feedback descriptivo en un modo constructivo pueden darle un impulso hacia la buena práctica docente.

El instrumento y el ejemplo que se han propuesto en este artículo pretenden facilitar la tarea del profesorado y contribuir a su formación docente por medio de la evaluación formativa y la observación sistemática sobre el uso de la lengua en el aula bilingüe.

\section{REFERENCIAS BIBLIOGRÁFICAS}

Bell, A., \& Mladenovic, R., (2008). The benefits of peer observation of teaching for tutor development. Higher Education, 55(6), 735-752.

Berman, E. (2003). A Short Guide to Evaluating Teaching. University of Arizona. Recuperado de http://www.tlataskforce.uconn.edu/docs/resources/A_Short_Guide_to_Evaluating_Teaching. pdf

Byrne, J., Brown, H., \& Challen, D., (2010). Peer development as an alternative to peer observation: A tool to enhance professional development. International Journal for Academic Development, 15(3), 215-228.

Clegg, J. (2007). Analyzing the Language Demands of Lessons Taught in a Second Language. 
Revista Española de Lingüística Aplicada (RESLA), 20(1), 113-128.

Consejería de Educación de la Junta de Andalucía. (2013). Guía Informativa para Centros de Enseñanza Bilingüe (2a ed.). Recuperado de http://www.juntadeandalucia.es/export/drupaljda/ Guia_informativa_centros_ense\%C3\%B1anza_bilingue_.pdf

Coyle, D., Hood, P., \& Marsh, D. (2010). Content and Language Integrated Learning. Cambridge: Cambridge University Press.

Dalton-Puffer, C. (2007). Discourse in Content and Language Integrated Learning (CLIL). Amsterdam: John Benjamins Publishing.

Engin, M., \& Priest, B. (2014). Observing Teaching: A Lens for Self-reflection. Journal of Perspectives in Applied Academic Practice, 2(2), 2-9.

Kearney, S., Perkins, T., \& Kennedy-Clark, S. (2016). Using self- and peer-assessments for summative purposes: Analyzing the relative validity of the AASL (authentic assessment for sustainable learning) model. Assessment and Evaluation in Higher Education, 41, 840-853.

Halbach, A. (2009). The primary school teacher and the challenges of bilingual education. In E. Dafouz \& M. Guerrini (Eds.), CLIL across educational levels (pp. 19-26). Madrid: Santillana.

Hamilton, R. (2012). His ideas are in my head: peer-to-peer teacher observations as professional development. Professional Development in Education, 39(1), 42-64. doi:10.1080/19415257.2 012.726202

Harper, F., \& Nicolson, M (2012). Online peer observation: its value in teacher professional development, support and well-being. International Journal for Academic Development, 18(3), 264-275. doi:10.1080/1360144X.2012.682159

Howard, A., \& Donaghue, H. (Eds). (2015). Teacher evaluation in second language education. London: Bloomsbury.

López-Pastor, V. M. (2012). Evaluación Formativa y Compartida en la Universidad: clarificación de conceptos y propuestas de intervención desde la Red Interuniversitaria de Evaluación Formativa. Psychology, Society \& Education, 4(1), 113-126.

Martín del Pozo, M.A. (2015). Teacher education for content and language integrated learning: insights from a current European debate. Revista Electrónica Interuniversitaria de Formación de Profesorado, 18(3), 153-168.

Mortaz Hejri, S., Mirzazadeh, A., \& Jalili, M. (2018). Peer observation of teaching for formative evaluation of faculty members. Med Education, 12(1), 12-16.

Nikula, T., Dafouz, E., Moore, P., \& Smit, U. (2016). Conceptualising Integration in CLIL and Multilingual Education. Bristol (UK): Multilingual Matters.

Pavesi, M., Bertochi, B., Hofmannová, M., \& Kazianka, M. (2001). Enseñar en una lengua extranjera: Cómo utilizar lenguas extranjeras en la enseñanza de una asignatura. Recuperado de http://www.ub.edu/filoan/CLIL/profesores.pdf

Pérez Cañado, M. L. (2014). Teacher training needs for bilingual education: In-service teacher perceptions. International Journal of Bilingual Education and Bilingualism, 19(3), 266-295. doi:0.1080/13670050.2014.980778

Pérez Cañado, M. L. (2016). Are teachers ready for CLIL? Evidence from a European study. European Journal of Teacher Education, 39(2), 202-221. doi:10.1080/02619768.2016.1138104

Sullivan, P., Buckle, A., Gregg, N., \& Atkinson, S. (2012). Peer Observation of teaching as a faculty development tool. BMC Medical Education, 1, 12-26. 